TRANSACTIONS OF THE

AMERICAN MATHEMATICAL SOCIETY

Volume 357, Number 2, Pages 473-487

S 0002-9947(04)03661-X

Article electronically published on May 28, 2004

\title{
ON THE BEHAVIOR OF THE ALGEBRAIC TRANSFER
}

\author{
ROBERT R. BRUNER, LÊ M. HÀ, AND NGUYỄN H. V. HƯNG
}

Dedicated to Professor Huỳnh Mùi on the occasion of his sixtieth birthday

Abstract. Let $\operatorname{Tr}_{k}: \mathbb{F}_{2} \underset{G L_{k}}{\otimes} P H_{i}\left(B \mathbb{V}_{k}\right) \rightarrow E x t_{\mathcal{A}}^{k, k+i}\left(\mathbb{F}_{2}, \mathbb{F}_{2}\right)$ be the algebraic transfer, which is defined by W. Singer as an algebraic version of the geometrical transfer $\operatorname{tr}_{k}: \pi_{*}^{S}\left(\left(B \mathbb{V}_{k}\right)_{+}\right) \rightarrow \pi_{*}^{S}\left(S^{0}\right)$. It has been shown that the algebraic transfer is highly nontrivial and, more precisely, that $T r_{k}$ is an isomorphism for $k=1,2,3$. However, Singer showed that $\operatorname{Tr}_{5}$ is not an epimorphism. In this paper, we prove that $T r_{4}$ does not detect the nonzero element $g_{s} \in \operatorname{Ext}_{\mathcal{A}}^{4,12 \cdot 2^{s}}\left(\mathbb{F}_{2}, \mathbb{F}_{2}\right)$ for every $s \geq 1$. As a consequence, the localized $\left(S q^{0}\right)^{-1} \operatorname{Tr}_{4}$ given by inverting the squaring operation $S q^{0}$ is not an epimorphism. This gives a negative answer to a prediction by Minami.

\section{INTRODUCTION AND STATEMENT OF RESULTS}

The subject of the present paper is the algebraic transfer

$$
T r_{k}: \mathbb{F}_{2} \underset{G L_{k}}{\otimes} P H_{i}\left(B \mathbb{V}_{k}\right) \rightarrow \operatorname{Ext}_{\mathcal{A}}^{k, k+i}\left(\mathbb{F}_{2}, \mathbb{F}_{2}\right),
$$

which is defined by W. Singer as an algebraic version of the geometrical transfer $\operatorname{tr}_{k}: \pi_{*}^{S}\left(\left(B \mathbb{V}_{k}\right)_{+}\right) \rightarrow \pi_{*}^{S}\left(S^{0}\right)$ to the stable homotopy groups of spheres. Here $\mathbb{V}_{k}$ denotes a $k$-dimensional $\mathbb{F}_{2}$-vector space, and $P H_{*}\left(B \mathbb{V}_{k}\right)$ is the primitive part consisting of all elements in $H_{*}\left(B \mathbb{V}_{k}\right)$ that are annihilated by every positive-degree operation in the mod 2 Steenrod algebra, $\mathcal{A}$. Throughout the paper, the homology is taken with coefficients in $\mathbb{F}_{2}$.

It has been proved that $\operatorname{Tr}_{k}$ is an isomorphism for $k=1,2$ by Singer [14] and for $k=3$ by Boardman [1]. These data together with the fact that $\operatorname{Tr}=$ $\bigoplus_{k \geq 0} \operatorname{Tr}_{k}$ is an algebra homomorphism (see [14]) show that $T r_{k}$ is highly nontrivial. Therefore, the algebraic transfer is considered to be a useful tool for studying the mysterious cohomology of the Steenrod algebra, $\operatorname{Ext}_{\mathcal{A}}^{*, *}\left(\mathbb{F}_{2}, \mathbb{F}_{2}\right)$. In [14], Singer also gave computations to show that $\operatorname{Tr}_{4}$ is an isomorphism up to a range of internal degrees. However, he proved that $\operatorname{Tr}_{5}$ is not an epimorphism.

Based on these data, we are particularly interested in the behavior of the fourth algebraic transfer. The following theorem is the main result of this paper.

Received by the editors June 18, 2003.

2000 Mathematics Subject Classification. Primary 55P47, 55Q45, 55S10, 55T15.

Key words and phrases. Adams spectral sequences, Steenrod algebra, invariant theory, algebraic transfer.

The third author was supported in part by the Vietnam National Research Program, Grant $\mathrm{N}^{0} 140801$. The computer calculations herein were done on equipment supplied by NSF grant DMS-0079743. 
Theorem 1.1. For each $s \geq 1$, the nonzero element $g_{s} \in \operatorname{Ext}_{\mathcal{A}}^{4,12 \cdot 2^{s}}\left(\mathbb{F}_{2}, \mathbb{F}_{2}\right)$ is not in the image of $\operatorname{Tr}_{4}$.

The reader is referred to May [11] for the generator $g_{1}$ and to Lin [8] or [9] for the generators $g_{s}$.

As a consequence, we get a negative answer to a prediction by Minami 13].

Corollary 1.2. The localization of the fourth algebraic transfer

$$
\left(S q^{0}\right)^{-1} \operatorname{Tr}_{4}:\left(S q^{0}\right)^{-1} \mathbb{F}_{2} \underset{G L_{4}}{\otimes} P H_{*}\left(B \mathbb{V}_{4}\right) \rightarrow\left(S q^{0}\right)^{-1} \operatorname{Ext}_{\mathcal{A}}^{4,4+*}\left(\mathbb{F}_{2}, \mathbb{F}_{2}\right)
$$

given by inverting $S q^{0}$ is not an epimorphism.

It is well known (see [10]) that there are squaring operations $S q^{i}(i \geq 0)$ acting on the cohomology of the Steenrod algebra, which share most of the properties with $S q^{i}$ on the cohomology of spaces. However, $S q^{0}$ is not the identity. We refer to Section 2 for the precise meaning of the operation $S q^{0}$ on the domain of the algebraic transfer.

We next explain the idea of the proof of Theorem 1.1

Let $P_{k}:=H^{*}\left(B \mathbb{V}_{k}\right)$ be the polynomial algebra of $k$ variables, each of degree 1 . Then, the domain of $\operatorname{Tr}_{k}, \mathbb{F}_{2} \otimes P H_{*}\left(B \mathbb{V}_{k}\right)$, is dual to $\left(\mathbb{F}_{2} \otimes P_{k}\right)^{G L_{k}}$. In order to prove Theorem 1.1, it suffices to show that $\left(\mathbb{F}_{2} \underset{\mathcal{A}}{\otimes P_{4}}\right)_{12 \cdot 2^{s}-4}^{G L_{4}} \stackrel{\mathcal{A}}{=} 0$, for every $s \geq 1$.

Direct calculation of $\left(\mathbb{F}_{2} \otimes P_{\mathcal{A}}\right)_{12 \cdot 2^{s}-4}$ is difficult, as $P_{4}$ in degree $12 \cdot 2^{s}-4$ is a huge $\mathbb{F}_{2}$-vector space, e.g. $\mathcal{A}^{\mathcal{A}}$ its dimension is 1771 for $s=1$. To compute it, we observe that the iterated dual squaring operation

$$
\left(S q_{*}^{0}\right)^{s}: \underset{\mathcal{A}}{\left(\mathbb{F}_{2} \otimes P_{4}\right)_{12 \cdot 2^{s}-4}} \rightarrow\left(\mathbb{F}_{2} \otimes P_{\mathcal{A}} P_{4}\right)_{8}
$$

is an isomorphism of $G L_{4}$-modules for any $s \geq 1$. This isomorphism is obtained by applying repeatedly the following proposition.

Proposition 1.3. Let $k$ and $r$ be positive integers. Suppose that each monomial $x_{1}^{i_{1}} \cdots x_{k}^{i_{k}}$ of $P_{k}$ in degree $2 r+k$ with at least one exponent $i_{t}$ even is hit. Then

$$
S q_{*}^{0}:\left(\mathbb{F}_{2} \underset{\mathcal{A}}{\otimes} P_{k}\right)_{2 r+k} \rightarrow\left(\mathbb{F}_{2} \underset{\mathcal{A}}{\otimes} P_{k}\right)_{r}
$$

is an isomorphism of $G L_{k}$-modules.

Here, as usual, we say that a polynomial $Q$ in $P_{k}$ is hit if it is $\mathcal{A}$-decomposable.

Further, we show that $\left(\mathbb{F}_{2} \otimes P_{4}\right)_{8}$ is an $\mathbb{F}_{2}$-vector space of dimension 55 . Then, by investigating a specific basis of it, we prove that $\left(\mathbb{F}_{2} \otimes_{\mathcal{A}} P_{4}\right)_{8}^{G L_{4}}=0$. As a consequence, we get $\left(\mathbb{F}_{2} \otimes P_{4}\right)_{12 \cdot 2^{s}-4}^{G L_{4}}=0$ for every $s \geq 1$.

The reader who does not wish to follow the invariant theory computation above may be satisfied by the following weaker theorem, and then would not need to read the paper's last 3 sections.

Theorem 1.4. $\mathrm{Tr}_{4}$ is not an isomorphism.

This theorem is proved by observing that, on the one hand,

$$
\left.\left(\mathbb{F}_{2} \underset{\mathcal{A}}{\otimes} P_{4}\right)_{20}^{G L_{4}} \cong \underset{\mathcal{A}}{\left(\mathbb{F}_{2} \otimes P_{4}\right.}\right)_{8}^{G L_{4}}
$$


and on the other hand,

$$
\operatorname{Ext}_{\mathcal{A}}^{4,4+20}\left(\mathbb{F}_{2}, \mathbb{F}_{2}\right)=\mathbb{F}_{2} \cdot g_{1} ¥ \operatorname{Ext}_{\mathcal{A}}^{4,4+8}\left(\mathbb{F}_{2}, \mathbb{F}_{2}\right)=0 .
$$

The paper is divided into six sections and organized as follows. Section 2 starts with a recollection of the squaring operation and ends with a proof of the isomorphism $\left(\mathbb{F}_{2} \otimes P_{4}\right)_{12 \cdot 2^{s}-4} \cong\left(\mathbb{F}_{2} \otimes P_{k}\right)_{8}$. Theorem 1.4 is proved in Section 3. We compute $\left(\mathbb{F}_{2} \otimes \mathcal{A}^{\mathcal{P}}{ }_{4}\right)_{8}$ and its $G L_{4}$-invariants in Section 4 . We prove Theorem[1.1]in Section 5. Finally, in Section 6, we describe the $G L_{4}$-module structure of $\left(\mathbb{F}_{2} \otimes{ }_{\mathcal{A}} P_{4}\right)_{8}$.

\section{A SUFFICIENT CONDITION FOR THE SQUARING OPERATION TO BE AN ISOMORPHISM}

This section starts with a recollection of Kameko's squaring operation

$$
S q^{0}: \mathbb{F}_{2} \underset{G L_{k}}{\otimes} P H_{*}\left(B \mathbb{V}_{k}\right) \rightarrow \mathbb{F}_{2} \otimes \underset{G L_{k}}{\otimes} P H_{*}\left(B \mathbb{V}_{k}\right)
$$

The most important property of Kameko's $S q^{0}$ is that it commutes with the classical $S q^{0}$ on $\operatorname{Ext}_{\mathcal{A}}^{*}\left(\mathbb{F}_{2}, \mathbb{F}_{2}\right)$ (defined in [10]) through the algebraic transfer (see [1], [13]).

This squaring operation is constructed as follows.

As is well known, $H^{*}\left(B \mathbb{V}_{k}\right)$ is the polynomial algebra, $P_{k}:=\mathbb{F}_{2}\left[x_{1}, \ldots, x_{k}\right]$, on $k$ generators $x_{1}, \ldots, x_{k}$, each of degree 1 . By dualizing,

$$
H_{*}\left(B \mathbb{V}_{k}\right)=\Gamma\left(a_{1}, \ldots, a_{k}\right)
$$

is the divided power algebra generated by $a_{1}, \ldots, a_{k}$, each of degree 1 , where $a_{i}$ is dual to $x_{i} \in H^{1}\left(B \mathbb{V}_{k}\right)$. Here the duality is taken with respect to the basis of $H^{*}\left(B \mathbb{V}_{k}\right)$ consisting of all monomials in $x_{1}, \ldots, x_{k}$.

In [6] and [7] Kameko defined a homomorphism

$$
\begin{aligned}
S q^{0}: \quad H_{*}\left(B \mathbb{V}_{k}\right) & \rightarrow H_{*}\left(B \mathbb{V}_{k}\right), \\
a_{1}^{\left(i_{1}\right)} \cdots a_{k}^{\left(i_{k}\right)} & \mapsto a_{1}^{\left(2 i_{1}+1\right)} \cdots a_{k}^{\left(2 i_{k}+1\right)},
\end{aligned}
$$

where $a_{1}^{\left(i_{1}\right)} \cdots a_{k}^{\left(i_{k}\right)}$ is dual to $x_{1}^{i_{1}} \cdots x_{k}^{i_{k}}$. The following lemma is well known. We give a proof to make the paper self-contained.

Lemma 2.1. $S q^{0}$ is a $G L_{k}$-homomorphism.

Proof. We use the explanation of $S q^{0}$ by Crabb and Hubbuck [3], which does not depend on the chosen basis of $H_{*}\left(B \mathbb{V}_{k}\right)$. The element $a\left(\mathbb{V}_{k}\right)=a_{1} \cdots a_{k}$ is nothing but the image of the generator of $\Lambda^{k}\left(\mathbb{V}_{k}\right)$ under the (skew) symmetrization map

$$
\Lambda^{k}\left(\mathbb{V}_{k}\right) \rightarrow H_{k}\left(B \mathbb{V}_{k}\right)=\Gamma_{k}\left(\mathbb{V}_{k}\right)=(\underbrace{\mathbb{V}_{k} \otimes \cdots \otimes \mathbb{V}_{k}}_{k \text { times }})_{S_{k}}
$$

where the symmetric group $S_{k}$ acts on $\mathbb{V}_{k} \otimes \cdots \otimes \mathbb{V}_{k}$ by permutations of the factors. Let $c: H_{*}\left(B \mathbb{V}_{k}\right) \rightarrow H_{*}\left(B \mathbb{V}_{k}\right)$ be the degree-halving epimorphism, which is dual to the Frobenius monomorphism $F: H^{*}\left(B \mathbb{V}_{k}\right) \rightarrow H^{*}\left(B \mathbb{V}_{k}\right)$ defined by $F(x)=x^{2}$ for any $x$. We have

$$
S q^{0}(c(y))=a\left(\mathbb{V}_{k}\right) y
$$

for $y \in H_{*}\left(B \mathbb{V}_{k}\right)$. To prove that this is well defined we need to show that if $c(y)=0$, then $a\left(\mathbb{V}_{k}\right) y=0$. Indeed, $c(y)=0$ implies $\langle c(y), x\rangle=\left\langle y, x^{2}\right\rangle=0$ for every $x \in H^{*}\left(B \mathbb{V}_{k}\right)$. Here $\langle\cdot, \cdot\rangle$ denotes the dual pairing between $H_{*}\left(B \mathbb{V}_{k}\right)$ and 
$H^{*}\left(B \mathbb{V}_{k}\right)$. So, if we write $y=\sum a_{1}^{\left(i_{1}\right)} \cdots a_{k}^{\left(i_{k}\right)}$, then there is at least one $i_{t}$ which is odd in each term of the sum. Therefore,

$$
a\left(\mathbb{V}_{k}\right) y=a_{1} \cdots a_{k}\left(\sum a_{1}^{\left(i_{1}\right)} \cdots a_{k}^{\left(i_{k}\right)}\right)=0,
$$

because $a_{t} a_{t}^{\left(i_{t}\right)}=0$ for any odd $i_{t}$. So, $S q^{0}$ is well defined.

As $c$ is a $G L_{k}$-epimorphism, the map $S q^{0}$ is a $G L_{k^{-}}$-homomorphism.

The lemma is proved.

Further, it is easy to see that $c S q_{*}^{2 t+1}=0, c S q_{*}^{2 t}=S q_{*}^{t} c$. So we have

$$
S q_{*}^{2 t+1} S q^{0}=0, S q_{*}^{2 t} S q^{0}=S q^{0} S q_{*}^{t} .
$$

(See 4] for an explicit proof.) Therefore, $S q^{0}$ maps $P H_{*}\left(B \mathbb{V}_{k}\right)$ to itself.

Kameko's $S q^{0}$ is defined by

$$
S q^{0}=1 \underset{G L_{k}}{\otimes} S q^{0}: \mathbb{F}_{2} \underset{G L_{k}}{\otimes} P H_{*}\left(B \mathbb{V}_{k}\right) \rightarrow \underset{G L_{k}}{\otimes} P H_{*}\left(B \mathbb{V}_{k}\right) .
$$

The dual homomorphism $S q_{*}^{0}: P_{k} \rightarrow P_{k}$ of $S q^{0}$ is obviously given by

$$
S q_{*}^{0}\left(x_{1}^{j_{1}} \cdots x_{k}^{j_{k}}\right)= \begin{cases}x_{1}^{\frac{j_{1}-1}{2}} \cdots x_{k}^{\frac{j_{k}-1}{2}}, & j_{1}, \ldots, j_{k} \text { odd }, \\ 0, & \text { otherwise. }\end{cases}
$$

Hence

$$
\operatorname{Ker}\left(S q_{*}^{0}: P_{k} \rightarrow P_{k}\right)=\overline{E v e n}
$$

where $\overline{\text { Even }}$ denotes the vector subspace of $P_{k}$ spanned by all monomials $x_{1}^{i_{1}} \cdots x_{k}^{i_{k}}$ with at least one exponent $i_{t}$ even.

Let $s: P_{k} \rightarrow P_{k}$ be a right inverse of $S q_{*}^{0}$ defined as follows:

$$
s\left(x_{1}^{i_{1}} \cdots x_{k}^{i_{k}}\right)=x_{1}^{2 i_{1}+1} \cdots x_{k}^{2 i_{k}+1} .
$$

It should be noted that $s$ does not commute with the doubling map on $\mathcal{A}$, that is, in general

$$
S q^{2 t} s \neq s S q^{t}
$$

However, in one particular circumstance we have the following.

Lemma 2.2. Under the hypothesis of Proposition 1.3, the map

$$
\begin{aligned}
\bar{s}:\left(\mathbb{F}_{2} \otimes P_{k}\right)_{r} & \rightarrow\left(\mathbb{F}_{2} \otimes P_{k}\right)_{2 r+k}, \\
\bar{s}[X] & =[s X]
\end{aligned}
$$

is a well-defined linear map.

Proof. We start with an observation that

$$
\operatorname{Im}\left(S q^{2 t} s-s S q^{t}\right) \subset \overline{\text { Even }} .
$$

We prove this by showing equivalently that

$$
S q_{*}^{0}\left(S q^{2 t} s-s S q^{t}\right)=0 .
$$

Indeed,

$$
\begin{aligned}
S q_{*}^{0}\left(S q^{2 t} s-s S q^{t}\right) & =S q_{*}^{0} S q^{2 t} s-S q_{*}^{0} s S q^{t} \\
& =S q^{t} S q_{*}^{0} s-S q_{*}^{0} s S q^{t} \\
& =S q^{t} \cdot i d-i d \cdot S q^{t} \\
& =0 .
\end{aligned}
$$


As a consequence, $s$ maps $\left(\mathcal{A}^{+} P_{k}\right)_{r}$ to $\left(\mathcal{A}^{+} P_{k}+\overline{\text { Even }}\right)_{2 r+k}$. Here and in what follows, $\mathcal{A}^{+}$denotes the submodule of $\mathcal{A}$ consisting of all positive degree operations. Further, by the hypothesis of Proposition 2.3, we have

$$
\left(\mathcal{A}^{+} P_{k}+\overline{\text { Even }}\right)_{2 r+k} \subset\left(\mathcal{A}^{+} P_{k}\right)_{2 r+k} .
$$

Hence, $s$ maps $\left(\mathcal{A}^{+} P_{k}\right)_{r}$ to $\left(\mathcal{A}^{+} P_{k}\right)_{2 r+k}$. So the map $\bar{s}$ is well defined. Then it is a linear map, as $s$ is.

The lemma is proved.

The following proposition is also numbered as Proposition 1.3.

Proposition 2.3. Let $k$ and $r$ be positive integers. Suppose that each monomial $x_{1}^{i_{1}} \cdots x_{k}^{i_{k}}$ of $P_{k}$ in degree $2 r+k$ with at least one exponent $i_{t}$ even is hit. Then

$$
S q_{*}^{0}:\left(\mathbb{F}_{2} \underset{\mathcal{A}}{\otimes} P_{k}\right)_{2 r+k} \rightarrow\left(\mathbb{F}_{2} \underset{\mathcal{A}}{\otimes} P_{k}\right)_{r}
$$

is an isomorphism of $G L_{k}$-modules.

Proof. On the one hand, we have $S q_{*}^{0} \bar{s}=i d_{\left(\mathbb{F}_{2} \otimes_{\mathcal{A}} P_{k}\right)_{r}}$. Indeed, from $S q_{*}^{0} s=i d_{P_{k}}$, it follows that

$$
S q_{*}^{0} \bar{s}[X]=S q_{*}^{0}[s X]=\left[S q_{*}^{0} s X\right]=[X],
$$

for any $X$ in degree $r$ of $P_{k}$.

On the other hand, we have $\bar{s} S q_{*}^{0}=i d_{\left(\mathbb{F}_{2} \otimes P_{k}\right)_{2 r+k}}$. Indeed, by the hypothesis, any monomial with at least one even $\mathcal{A}$ exponent represents the 0 class in $\left(\mathbb{F}_{2} \otimes P_{k}\right)_{2 r+k}$, so we need only to check on the classes of monomials with all exponents odd. We have

$$
\begin{aligned}
\bar{s} S q_{*}^{0}\left[x_{1}^{2 i_{1}+1} \cdots x_{k}^{2 i_{k}+1}\right] & =\bar{s}\left[x_{1}^{i_{1}} \cdots x_{k}^{i_{k}}\right] \\
& =\left[s\left(x_{1}^{i_{1}} \cdots x_{k}^{i_{k}}\right)\right] \\
& =\left[x_{1}^{2 i_{1}+1} \cdots x_{k}^{2 i_{k}+1}\right],
\end{aligned}
$$

for any $x_{1}^{2 i_{1}+1} \cdots x_{k}^{2 i_{k}+1}$ in degree $2 r+k$ of $P_{k}$.

Combining the two equalities, $S q_{*}^{0} \bar{s}=i d_{\left(\mathbb{F}_{2} \otimes P_{k}\right)_{r}}$ and $\bar{s} S q_{*}^{0}=i d_{\left(\mathbb{F}_{2} \otimes P_{k}\right)_{2 r+k}}$, we see that $S q_{*}^{0}:\left(\mathbb{F}_{2} \otimes P_{k}\right)_{2 r+k} \rightarrow\left(\mathbb{F}_{2} \otimes P_{k}\right)_{r}$ is an isomorphism with inverse $\bar{s}$ : $\left(\mathbb{F}_{2} \otimes P_{\mathcal{A}}\right)_{r} \rightarrow\left(\mathbb{F}_{2} \otimes P_{\mathcal{A}}\right)_{2 r+k}^{\mathcal{A}}$

The proposition is proved.

The target of this section is the following.

Lemma 2.4. For every positive integer $s$,

$$
\left(S q_{*}^{0}\right)^{s}: \underset{\mathcal{A}}{\left(\mathbb{F}_{2} \otimes P_{4}\right)_{12 \cdot 2^{s}-4}} \rightarrow\left(\mathbb{F}_{2} \underset{\mathcal{A}}{\otimes} P_{4}\right)_{8}
$$

is an isomorphism of $G L_{4}$-modules.

Proof. By using Proposition 2.3 repeatedly, it suffices to show that any monomial of $P_{4}$ in degree $m=12 \cdot 2^{s}-4$ with at least one even exponent is hit. Since $m$ is even, the number of even exponents in such a monomial must be either 2 or 4 . If all exponents of the monomial are even, then it is hit by $S q^{1}$. Hence we need only to consider the case of a monomial $R$ with exactly two even exponents (and so exactly two odd exponents). Wood proves ([15]) that if $\alpha\left(m+\alpha_{0}(R)\right)>\alpha_{0}(R)$ 
then $R$ is hit, where $\alpha_{0}(R)$ is the number of odd exponents in the monomial $R$, and $\alpha(n)$ is the number of ones in the binary expansion of $n$. We have $\alpha_{0}(R)=2$ and $\alpha\left(m+\alpha_{0}(R)\right)=\alpha\left(12 \cdot 2^{s}-2\right)=s+2$, so Wood's criterion is met, and $R$ is hit.

The lemma is proved.

\section{ThE FOURTH ALGEBRAIC TRANSFER IS NOT AN ISOMORPHISM}

The target of this section is to prove the following theorem, which is also numbered as Theorem 1.4 .

\section{Theorem 3.1.}

$$
\operatorname{Tr}_{4}: \mathbb{F}_{2} \underset{G L_{4}}{\otimes} P H_{i}\left(B \mathbb{V}_{4}\right) \rightarrow \operatorname{Ext}_{\mathcal{A}}^{4,4+i}\left(\mathbb{F}_{2}, \mathbb{F}_{2}\right)
$$

is not an isomorphism.

Proof. For any $r$, we have a commutative diagram

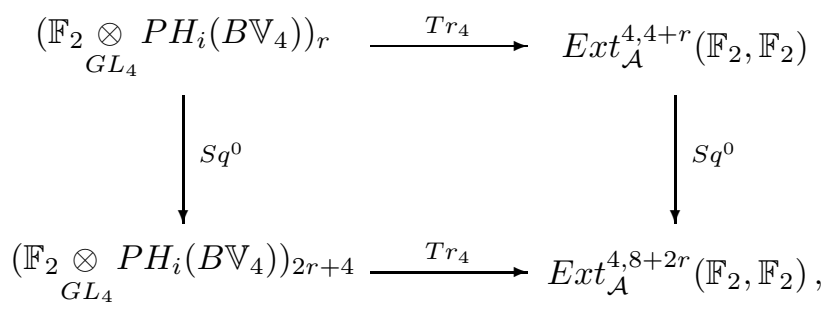

where the first vertical arrow is the Kameko $S q^{0}$ and the second vertical one is the classical $S q^{0}$.

The dual statement of Lemma 2.4 for $s=2$ claims that

$$
\left.S q^{0}: \underset{G L_{4}}{\left(\mathbb{F}_{2} \otimes\right.} P H_{i}\left(B \mathbb{V}_{4}\right)\right)_{8} \rightarrow\left(\mathbb{F}_{2} \underset{G L_{4}}{\otimes} P H_{i}\left(B \mathbb{V}_{4}\right)\right)_{20}
$$

is an isomorphism. On the other hand, it is known (May [11]) that

$$
\operatorname{Ext}_{\mathcal{A}}^{4,4+8}\left(\mathbb{F}_{2}, \mathbb{F}_{2}\right)=0 \approx \operatorname{Ext}_{\mathcal{A}}^{4,4+20}\left(\mathbb{F}_{2}, \mathbb{F}_{2}\right)=\mathbb{F}_{2} \cdot g_{1} \text {. }
$$

This implies that $\mathrm{Tr}_{4}$ is not an isomorphism. The theorem is proved.

Remark 3.2. This proof does not show whether $T r_{4}$ fails to be a monomorphism or fails to be an epimorphism. We will see that actually $T r_{4}$ is not an epimorphism in Section 5 below.

\section{4. $G L_{4}$-INVARIANts of THE IndeComposables of $P_{4}$ IN DEGREE 8}

From now on, let us write $x=x_{1}, y=x_{2}, z=x_{3}$ and $t=x_{4}$ and denote the monomial $x^{a} y^{b} z^{c} t^{d}$ by $(a, b, c, d)$ for abbreviation.

Proposition 4.1. $\left(\mathbb{F}_{2} \otimes P_{4}\right)_{8}$ is an $\mathbb{F}_{2}$-vector space of dimension 55 with a basis consisting of the classes represented by the following monomials:

(A) $(7,1,0,0),(7,0,1,0),(7,0,0,1),(1,7,0,0),(1,0,7,0),(1,0,0,7)$, $(0,7,1,0),(0,7,0,1),(0,1,7,0),(0,1,0,7),(0,0,7,1),(0,0,1,7)$,

(B) $(3,3,1,1),(3,1,3,1),(3,1,1,3),(1,3,3,1),(1,3,1,3),(1,1,3,3)$, 
(C) $(6,1,1,0),(6,1,0,1),(6,0,1,1),(1,6,1,0),(1,6,0,1),(1,1,6,0)$, $(1,1,0,6),(1,0,6,1),(1,0,1,6),(0,6,1,1),(0,1,6,1),(0,1,1,6)$,

(D) $(5,3,0,0),(5,0,3,0),(5,0,0,3),(0,5,3,0),(0,5,0,3),(0,0,5,3)$,

(E) $(5,2,1,0),(5,2,0,1),(5,0,2,1),(2,5,1,0),(2,5,0,1),(2,1,5,0)$, $(2,1,0,5),(2,0,5,1),(2,0,1,5),(0,5,2,1),(0,2,5,1),(0,2,1,5)$,

(F) $(5,1,1,1),(1,5,1,1),(1,1,5,1),(1,1,1,5)$,

(G) $(4,2,1,1),(4,1,2,1),(1,4,2,1)$.

The proposition is proved by combining a couple of lemmas.

Lemma 4.2. $\left(\mathbb{F}_{2} \otimes P_{\mathcal{A}}\right)_{8}$ is generated by the 55 elements listed in Proposition 4.1 . Proof. It is easy to see that every monomial $(a, b, c, d)$ with $a, b, c, d$ all even is hit (more precisely by $S q^{1}$ ).

The only monomials $(a, b, c, d)$ in degree 8 with at least one of $a, b, c, d$ odd are the following up to permutations of the variables:

$$
\begin{aligned}
& (7,1,0,0),(3,3,1,1),(6,1,1,0),(5,3,0,0),(5,2,1,0),(5,1,1,1),(4,2,1,1), \\
& (4,3,1,0),(3,3,2,0),(3,2,2,1) .
\end{aligned}
$$

The last 3 monomials and their permutations are expressed in terms of the first 7 monomials and their permutations as follows:

$$
\begin{aligned}
(4,3,1,0)= & (2,5,1,0)+S q^{4}(1,2,1,0)+S q^{2}(2,3,1,0) \\
(3,3,2,0)= & (5,2,1,0)+(2,5,1,0)+S q^{4}(2,1,1,0)+S q^{4}(1,2,1,0) \\
& +S q^{2}(3,2,1,0)+S q^{2}(2,3,1,0)+S q^{1}(3,3,1,0) \\
(3,2,2,1)= & (5,1,1,1)+(4,2,1,1)+(4,1,2,1) \\
& +S q^{2}(3,1,1,1)+S q^{1}(4,1,1,1)+S q^{1}(3,2,1,1)+S q^{1}(3,1,2,1) .
\end{aligned}
$$

Hence, $\left(\mathbb{F}_{2} \otimes P_{4}\right)_{8}$ is generated by the following 7 monomials and their permutations:

$$
(7,1,0,0),(3,3,1,1),(6,1,1,0),(5,3,0,0),(5,2,1,0),(5,1,1,1),(4,2,1,1) .
$$

By the family of a monomial $(a, b, c, d)$ we mean the set of all monomials which are obtained from $(a, b, c, d)$ by permutations of the variables.

The monomials in the 7 families above which are not in Proposition 4.1 can be expressed in terms of the 55 elements listed there as follows. (We give only one expression from each symmetry class.)

$$
\begin{aligned}
(3,5,0,0)= & (5,3,0,0)+S q^{4}(2,2,0,0)+S q^{2}(3,3,0,0) \\
(5,1,2,0)= & (6,1,1,0)+(5,2,1,0)+S q^{1}(5,1,1,0) \\
(4,1,1,2)= & (4,2,1,1)+(4,1,2,1)+S q^{1}(4,1,1,1) \\
(2,4,1,1)= & (4,2,1,1)+S q^{4}(1,1,1,1)+S q^{2}(2,2,1,1) \\
(2,1,1,4)= & (4,2,1,1)+(4,1,2,1) \\
& +S q^{4}(1,1,1,1)+S q^{2}(2,1,1,2)+S q^{1}(4,1,1,1)
\end{aligned}
$$




$$
\begin{aligned}
(1,4,1,2)= & (4,2,1,1)+(1,4,2,1) \\
& +S q^{4}(1,1,1,1)+S q^{2}(2,2,1,1)+S q^{1}(1,4,1,1) \\
(1,2,1,4)= & (4,2,1,1)+(1,4,2,1) \\
& +S q^{2}(2,2,1,1)+S q^{2}(1,2,1,2)+S q^{1}(1,4,1,1) \\
(1,1,4,2)= & (4,1,2,1)+(1,4,2,1) \\
& +S q^{2}(2,1,2,1)+S q^{2}(1,2,2,1)+S q^{1}(1,1,4,1) \\
(1,1,2,4)= & (4,1,2,1)+(1,4,2,1)+S q^{4}(1,1,1,1) \\
& +S q^{2}(2,1,2,1)+S q^{2}(1,2,2,1)+S q^{2}(1,1,2,2)+S q^{1}(1,1,4,1)
\end{aligned}
$$

The lemma is proved.

Lemma 4.3. The 55 elements listed in Proposition 4.1 are linearly independent in $\left(\mathbb{F}_{2} \otimes P_{\mathcal{A}}\right)_{8}$.

Proof. We will use an equivalence relation defined by saying that, for two polynomials $P$ and $Q, P$ is equivalent to $Q$, denoted by $P \sim Q$, if $P-Q$ is hit.

If $X$ is one of the letters from $A$ to $G$, let $X_{i}$ be the $i$-th element in family $X$ according to the order listed in Proposition 4.1 (This is the lexicographical order in each family.)

Suppose there is a linear relation between the 55 elements listed there,

$$
\sum_{i=1}^{12} a_{i} A_{i}+\sum_{i=1}^{6} b_{i} B_{i}+\sum_{i=1}^{12} c_{i} C_{i}+\sum_{i=1}^{6} d_{i} D_{i}+\sum_{i=1}^{12} e_{i} E_{i}+\sum_{i=1}^{4} f_{i} F_{i}+\sum_{i=1}^{3} g_{i} G_{i}=0,
$$

where $a_{i}, b_{i}, c_{i}, d_{i}, e_{i}, f_{i}, g_{i} \in \mathbb{F}_{2}$. We need to show that all these coefficients are zero. The proof is divided into 4 steps.

Step 1 . We call a monomial a spike if each of its exponents is of the form $2^{n}-1$ for some $n$. It is well known that spikes do not appear in the expression of $S q^{i} Y$ for any $i$ positive and any monomial $Y$, since the powers $x^{2^{n}-1}$ are not hit in the one variable case. Hence, the coefficient of any spike is zero in every linear relation in $\mathbb{F}_{2} \otimes P_{k}$.

Among the 55 elements of Proposition 4.1, the classes of families $A$ and $B$ are spikes. So $a_{i}=b_{j}=0$, for every $i$ and $j$. Then, we get

$$
\sum_{i=1}^{12} c_{i} C_{i}+\sum_{i=1}^{6} d_{i} D_{i}+\sum_{i=1}^{12} e_{i} E_{i}+\sum_{i=1}^{4} f_{i} F_{i}+\sum_{i=1}^{3} g_{i} G_{i}=0 .
$$

Step 2. Consider the homomorphism $\mathbb{F}_{2} \underset{\mathcal{A}}{\otimes} P_{4} \rightarrow \mathbb{F}_{2} \underset{\mathcal{A}}{\otimes} P_{2}$ induced by the projection $P_{4} \rightarrow P_{4} /(z, t) \cong P_{2}$. Under this homomorphism, the image of the above linear relation is $d_{1}(5,3)=0$.

In order to show that $d_{1}=0$, we need to prove that $(5,3)$ is nonzero in $\mathbb{F}_{2} \otimes P_{2}$. The linear transformation $x \mapsto x, y \mapsto x+y$ sends $(5,3)$ to $(8,0)+(7,1)+(6, \mathcal{A}, 2)+$ $(5,3) \sim(7,1)+(5,3)$. As the action of the Steenrod algebra commutes with linear maps, if $(5,3)$ is hit then so is $(7,1)+(5,3)$. But it is impossible, because $(7,1)$ is a spike. Hence, $(5,3) \neq 0$ in $\mathbb{F}_{2} \otimes P_{2}$ and $d_{1}=0$. 
Similarly, using all the projections of $P_{4}$ to its quotients by the ideals generated by each pair of the four variables, we get $d_{i}=0$ for every $i$. So we get

$$
\sum_{i=1}^{12} c_{i} C_{i}+\sum_{i=1}^{12} e_{i} E_{i}+\sum_{i=1}^{4} f_{i} F_{i}+\sum_{i=1}^{3} g_{i} G_{i}=0
$$

Step 3. Consider the homomorphism $\mathbb{F}_{2} \otimes P_{\mathcal{A}} \rightarrow \mathbb{F}_{2} \otimes P_{\mathcal{A}} P_{3}$ induced by the projection $P_{4} \rightarrow P_{4} /(t) \cong P_{3}$. Under this homomorphism, the linear relation above is sent to $c_{1}(6,1,1)+c_{4}(1,6,1)+c_{6}(1,1,6)+e_{1}(5,2,1)+e_{4}(2,5,1)+e_{6}(2,1,5)=0$.

Applying the linear map $x \mapsto x, y \mapsto x, z \mapsto y$ to this relation, we obtain

$$
\begin{aligned}
& \left(c_{1}+c_{4}+e_{1}+e_{4}\right)(7,1)+c_{6}(2,6)+e_{6}(3,5) \\
& \quad=\left(c_{1}+c_{4}+e_{1}+e_{4}\right)(7,1)+e_{6}(3,5)=0 .
\end{aligned}
$$

Since $(7,1)$ is a spike, $\left(c_{1}+c_{4}+e_{1}+e_{4}\right)=0$, hence $e_{6}(3,5)=0$. As for $(5,3)$, we can show that $(3,5) \neq 0 \in \mathbb{F}_{2} \otimes P_{2}$ and get $e_{6}=0$.

By similar arguments, we have $e_{1}=e_{4}=e_{6}=0$. The equality $\left(c_{1}+c_{4}+e_{1}+e_{4}\right)=$ 0 shows that $c_{1}+c_{4}=0$ or $c_{1}=c_{4}$. By similar arguments, $c_{1}=c_{4}=c_{6}$. We denote this common coefficient by $c$ and get

$$
c\{(6,1,1)+(1,6,1)+(1,1,6)\}=0 .
$$

We prove that $c=0$ by showing that $(6,1,1)+(1,6,1)+(1,1,6) \neq 0$. Suppose the contrary, that $(6,1,1)+(1,6,1)+(1,1,6)$ is hit. Then, by the unstable property of the action of $\mathcal{A}$ on the polynomial algebra, we have

$$
(6,1,1)+(1,6,1)+(1,1,6)=S q^{1}(P)+S q^{2}(Q)+S q^{4}(R),
$$

for some polynomials $P, Q, R$. By the degree information, $S q^{4}(R)=R^{2}$ and this element is hit by $S q^{1}$. Therefore, it suffices to assume $(6,1,1)+(1,6,1)+(1,1,6)=$ $S q^{1}(P)+S q^{2}(Q)$.

Let $S q^{2} S q^{2} S q^{2}$ act on the both sides of this equality. The right hand side is sent to zero, as $S q^{2} S q^{2} S q^{2}$ annihilates $S q^{1}$ and $S q^{2}$. On the other hand,

$$
S q^{2} S q^{2} S q^{2}\{(6,1,1)+(1,6,1)+(1,1,6)\}=(8,4,2)+\text { symmetries } \neq 0 .
$$

This is a contradiction. So, it implies $(6,1,1)+(1,6,1)+(1,1,6) \neq 0$ and $c=0$. We get

$$
\sum_{i=1}^{4} f_{i} F_{i}+\sum_{i=1}^{3} g_{i} G_{i}=0 .
$$

Step 4. Apply the linear map $x \mapsto x, y \mapsto y, z \mapsto y, t \mapsto y$ to the above equality, and we have

$$
\begin{gathered}
f_{1}(5,3)+\left(f_{2}+f_{3}+f_{4}+g_{3}\right)(1,7)+\left(g_{1}+g_{2}\right)(4,4) \\
=f_{1}(5,3)+\left(f_{2}+f_{3}+f_{4}+g_{3}\right)(1,7)=0 .
\end{gathered}
$$

As $(7,1)$ is a spike, we obtain $\left(f_{2}+f_{3}+f_{4}+g_{3}\right)=0$ and $f_{1}(5,3)=0$. As $(5,3) \neq 0$, it yields $f_{1}=0$.

Next, apply the linear map $x \mapsto x, y \mapsto y, z \mapsto x, t \mapsto x$ to the equality $\sum_{i \neq 1} f_{i} F_{i}+\sum_{i=1}^{3} g_{i} G_{i}=0$, and we have

$$
\begin{gathered}
f_{2}(3,5)+\left(f_{3}+f_{4}+g_{2}\right)(7,1)+g_{1}(6,2)+g_{3}(4,4) \\
=f_{2}(3,5)+\left(f_{3}+f_{4}+g_{2}\right)(7,1)=0 .
\end{gathered}
$$


As $(7,1)$ is a spike, we get $\left(f_{3}+f_{4}+g_{2}\right)=0$ and $f_{2}(3,5)=0$. Since $(3,5) \neq 0$, it implies $f_{2}=0$.

Similarly, apply the linear map $x \mapsto x, y \mapsto x, z \mapsto y, t \mapsto x$ to the equality $f_{3} F_{3}+f_{4} F_{4}+\sum_{i=1}^{3} g_{i} G_{i}=0$, and we have

$$
\begin{gathered}
f_{3}(3,5)+\left(f_{4}+g_{1}\right)(7,1)+\left(g_{2}+g_{3}\right)(6,2) \\
=f_{3}(3,5)+\left(f_{4}+g_{1}\right)(7,1)=0 .
\end{gathered}
$$

As $(7,1)$ is a spike, we get $f_{4}+g_{1}=0$ and then $f_{3}=0$.

Finally, apply the linear map $x \mapsto x, y \mapsto x, z \mapsto x, t \mapsto y$ to the equality $f_{4} F_{4}+\sum_{i=1}^{3} g_{i} G_{i}=0$, and we have

$$
\begin{aligned}
& f_{4}(3,5)+\left(g_{1}+g_{2}+g_{3}\right)(7,1) \\
& \quad=f_{4}(3,5)+\left(g_{1}+g_{2}+g_{3}\right)(7,1)=0 .
\end{aligned}
$$

As $(7,1)$ is a spike, we get $g_{1}+g_{2}+g_{3}=0$ and then $f_{4}=0$.

Substituting $f_{1}=f_{2}=f_{3}=f_{4}=0$ into the equations $\left(f_{2}+f_{3}+f_{4}+g_{3}\right)=0$, $\left(f_{3}+f_{4}+g_{2}\right)=0, f_{4}+g_{1}=0$, we get $g_{1}=g_{2}=g_{3}=0$.

We have shown that all coefficients of an arbitrary linear relation between the 55 elements listed in Proposition 4.1 are zero. The lemma follows.

Combining Lemmas 4.24.3, we get Proposition 4.1

Proposition 4.4. $\left(\mathbb{F}_{2} \otimes P_{\mathcal{A}}\right)_{8}^{G L_{4}}=0$.

Proof. If $X$ is one of the letters $A, B, C, D, E, F, G$, let $\mathcal{L}(X)$ be the vector subspace of $\left(\mathbb{F}_{2} \otimes P_{4}\right)_{8}$ spanned by the elements of family $X$ in Proposition 4.1 . Let $S_{k}$ denote the symmetric subgroup of $G L_{k}$. According to the relations listed in the proof of Lemma $4.2, \mathcal{L}(A), \mathcal{L}(B), \mathcal{L}(C), \mathcal{L}(D), \mathcal{L}(F), \mathcal{L}(G)$ are $S_{4}$-submodules. The subspace $\mathcal{L}(E)$ is not an $S_{4}$-submodule. However, the sum

$$
\mathcal{L}(C, E)=\mathcal{L}(C) \oplus \mathcal{L}(E)
$$

is. We have a decomposition of $S_{4}$-modules

$$
\left(\mathbb{F}_{2} \otimes_{\mathcal{A}} P_{4}\right)_{8}=\mathcal{L}(A) \oplus \mathcal{L}(B) \oplus \mathcal{L}(C, E) \oplus \mathcal{L}(D) \oplus \mathcal{L}(F) \oplus \mathcal{L}(G) .
$$

Let $\alpha$ be an arbitrary $G L_{4}$-invariant in $\left(\mathbb{F}_{2} \underset{\mathcal{A}}{\otimes} P_{4}\right)_{8}$. It can uniquely be written in the form

$$
\alpha=\alpha_{A}+\alpha_{B}+\alpha_{C, E}+\alpha_{D}+\alpha_{F}+\alpha_{G},
$$

where $\alpha_{X} \in \mathcal{L}(X)$ for $X \in\{A, B, D, F, G\}$, and $\alpha_{C, E} \in \mathcal{L}(C, E)$. Each term of this sum is $S_{4}$-invariant.

Note that if a linear combination of elements in a family is $S_{4}$-invariant, then all of its coefficients are equal, because each element in the family can be obtained from any other by a suitable permutation. Let $s_{X}$ denote the sum of all the elements in the family $X$ listed in Proposition 4.1 Then, we have $\alpha_{A}=a s_{A}, \alpha_{B}=b s_{B}$, $\alpha_{D}=d s_{D}, \alpha_{F}=f s_{F}, \alpha_{G}=g s_{G}$, and $\alpha_{C, E}=c s_{C}+e s_{E}$, where $a, b, c, d, e, f, g \in \mathbb{F}_{2}$.

Let $p$ be the transposition given by $p(x)=y, p(y)=x, p(z)=z, p(t)=t$. It is easy to see that

$$
\begin{aligned}
& p(2,1,0,5)=(1,2,0,5)=(2,1,0,5)+(1,1,0,6), \\
& p(2,1,5,0)=(1,2,5,0)=(2,1,5,0)+(1,1,6,0) .
\end{aligned}
$$


Further, the 10 elements different from $(2,1,0,5)$ and $(2,1,5,0)$ in family $E$ are divided into 5 pairs with $p$ acting on each pair by twisting. So, $p\left(s_{E}\right)=s_{E}+$ $(1,1,0,6)+(1,1,6,0)$. On the other hand, as the family $C$ is full, in the sense that it contains all the variable permutations of a monomial, we have $p\left(s_{C}\right)=s_{C}$. Hence, we get

$$
p\left(\alpha_{C, E}\right)=p\left(c s_{C}+e s_{E}\right)=c s_{C}+e s_{E}+e(1,1,0,6)+e(1,1,6,0) .
$$

As $\alpha_{C, E}$ is $S_{4}$-invariant, $e(1,1,0,6)+e(1,1,6,0)=0$. So $e=0$, because the two elements are linearly independent by Lemma 4.3. We obtain

$$
\alpha=\alpha_{A}+\alpha_{B}+\alpha_{C}+\alpha_{D}+\alpha_{F}+\alpha_{G},
$$

where $\alpha_{C}=\alpha_{C, E}=c s_{C}$.

Let us now consider the transvection $\varphi$ given by $\varphi(x)=x, \varphi(y)=y, \varphi(z)=z$, $\varphi(t)=x+t$. A routine computation shows

$$
\begin{aligned}
\varphi\left(s_{A}\right)= & s_{A}+(7,1,0,0)+(7,0,1,0)+(7,0,0,1)+(1,7,0,0)+(1,0,7,0) \\
& +(6,1,0,1)+(6,0,1,1)+(1,1,0,6)+(1,0,1,6), \\
\varphi\left(s_{B}\right)= & s_{B}+(6,1,1,0)+(1,6,1,0)+(1,1,6,0)+(2,5,1,0)+(2,1,5,0) \\
& +(5,1,1,1)+(1,5,1,1)+(1,1,5,1)+(4,2,1,1)+(4,1,2,1) \\
& +(3,3,1,1)+(3,1,3,1), \\
\varphi\left(s_{C}\right)= & s_{C}+(6,1,1,0)+(1,6,1,0)+(1,1,6,0), \\
\varphi\left(s_{D}\right)= & s_{D}+(7,0,0,1)+(1,6,0,1)+(1,0,6,1)+(5,3,0,0)+(5,0,3,0), \\
\varphi\left(s_{F}\right)= & s_{F}+(2,5,1,0)+(2,1,5,0)+(5,1,1,1)+(4,2,1,1)+(4,1,2,1), \\
\varphi\left(s_{G}\right)= & s_{G}+(6,1,1,0) .
\end{aligned}
$$

Let $r_{X}=\varphi\left(s_{X}\right)-s_{X}$ where $X$ is one of the letters $A, B, C, D, F, G$. The equality $\varphi(\alpha)=\alpha$ is rewritten as

$$
\varphi\left(a s_{A}+b s_{B}+c s_{C}+d s_{D}+f s_{F}+g s_{G}\right)=a s_{A}+b s_{B}+c s_{C}+d s_{D}+f s_{F}+g s_{G},
$$

or equivalently

$$
a r_{A}+b r_{B}+c r_{C}+d r_{D}+f r_{F}+g r_{G}=0
$$

In this linear combination, $r_{B}$ and $r_{D}$ are the only terms containing $(3,3,1,1)$ in family $B$ and $(5,3,0,0)$ in family $D$ respectively. From Lemma 4.3 , we get $b=d=0$, and therefore $a r_{A}+c r_{C}+f r_{F}+g r_{G}=0$.

In the new linear combination, as $r_{A}, r_{C}$ and $r_{F}$ are the only terms containing $(7,1,0,0)$ in family $A,(1,6,1,0)$ in family $C$ and $(4,2,1,1)$ in family $F$ respectively, we have $a=c=f=0$. As a consequence, $g r_{G}=0$, so we finally get $g=0$.

In summary, we have shown that every $G L_{4}$-invariant $\alpha$ in $\left(\mathbb{F}_{2} \underset{\mathcal{A}}{\otimes} P_{4}\right)_{8}$ equals zero. The proposition is proved.

\section{The FOURTh ALgebraiC TRANSFER IS NOT AN EPIMORPHISM}

The goal of this paper is to prove the following theorem, which is also numbered as Therem 1.1 .

Theorem 5.1. For each $s \geq 1$,

$$
\operatorname{Tr}_{4}: \mathbb{F}_{2} \underset{G L_{4}}{\otimes} P H_{i}\left(B \mathbb{V}_{4}\right) \rightarrow \operatorname{Ext}_{\mathcal{A}}^{4,4+i}\left(\mathbb{F}_{2}, \mathbb{F}_{2}\right)
$$

does not detect the nonzero element $g_{s} \in \operatorname{Ext}_{\mathcal{A}}^{4,12 \cdot 2^{s}}\left(\mathbb{F}_{2}, \mathbb{F}_{2}\right)$. 
Proof. Combining Lemma 2.4 and Proposition 4.4 we get

$$
\left(\mathbb{F}_{2} \underset{\mathcal{A}}{\otimes} P_{4}\right)_{12 \cdot 2^{s}-4}^{G L_{4}}=0
$$

for every nonnegative integer $s$.

On the other hand, it is well known that $\operatorname{Ext}_{\mathcal{A}}^{4,24}\left(\mathbb{F}_{2}, \mathbb{F}_{2}\right)$ is spanned by the generator $g_{1}$ (see May [11]). Further, $g_{s}=\left(S q^{0}\right)^{s-1}\left(g_{1}\right)$ is nonzero in $\operatorname{Ext}_{\mathcal{A}}^{4,12 \cdot 2^{s}}\left(\mathbb{F}_{2}, \mathbb{F}_{2}\right)$ (see Lin [8] and also [9]).

$$
\begin{aligned}
& \text { As } \mathbb{F}_{2} \underset{G L_{4}}{\otimes} P H_{12 \cdot 2^{s}-4}\left(B \mathbb{V}_{4}\right) \text { is dual to }\left(\mathbb{F}_{2} \underset{\mathcal{A}}{\otimes} P_{4}\right)_{12 \cdot 2^{s}-4}^{G L_{4}}, \\
& \qquad r_{4}: \mathbb{F}_{2} \underset{G L_{4}}{\otimes} P H_{12 \cdot 2^{s}-4}\left(B \mathbb{V}_{4}\right) \rightarrow \operatorname{Ext}_{\mathcal{A}}^{4,12 \cdot 2^{s}}\left(\mathbb{F}_{2}, \mathbb{F}_{2}\right)
\end{aligned}
$$

does not detect the generator $g_{s}$, for every nonnegative integer $s$.

The theorem is proved.

As a consequence, we get a negative answer to a prediction by Minami [13]. (This corollary is also numbered as Corollary [1.2)

Corollary 5.2. The localization of the fourth algebraic transfer

$$
\left(S q^{0}\right)^{-1} \operatorname{Tr}_{4}:\left(S q^{0}\right)^{-1} \mathbb{F}_{2} \underset{G L_{4}}{\otimes} P H_{*}\left(B \mathbb{V}_{4}\right) \rightarrow\left(S q^{0}\right)^{-1} \operatorname{Ext}_{\mathcal{A}}^{4,4+*}\left(\mathbb{F}_{2}, \mathbb{F}_{2}\right)
$$

given by inverting $S q^{0}$ is not an epimorphism.

Proof. Indeed, it does not detect the nonzero element $g$, which is represented by the family $\left(g_{s}\right)_{s>0}$ with $g_{s}=\left(S q^{0}\right)^{s-1}\left(g_{1}\right)$. The corollary follows.

Remark 5.3. Our result does not affect Singer's conjecture that the $k$-th algebraic transfer is a monomorphism for every $k$. (See [14].)

\section{Final Remark: $G L_{4}$-Module Structure}

Boardman's study of the 3 variable problem shows that the $G L_{k}$ module structure of $\mathbb{F}_{2} \otimes P_{k}$ may be a useful tool. In this vein we close with a description of the module $\left(\mathbb{F}_{2}^{\mathcal{A}} \otimes P_{4}\right)_{8}$ as a $G L_{4}$-module. From the "Modular Atlas" [5] we find that

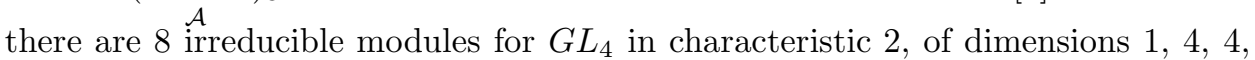
$6,14,20,20$, and 64 . With a little calculation we find the following description of them:

1: the trivial module $\mathbb{F}_{2}$,

$N$ : the natural module $\mathbb{F}_{2}^{4}$,

$N^{*}$ : the dual of the natural module,

$\Lambda$ : the alternating square of $N$ or $N^{*}$,

$S$ : the nontrivial constituent of $N \otimes N^{*}$, which has composition factors $1, S, 1$,

$T$ : a constituent of $N \otimes \Lambda$, which has composition factors $N^{*}$ and $T$,

$T^{*}$ : a constituent of $N^{*} \otimes \Lambda$, which has composition factors $N$ and $T^{*}$,

$S t$ : the Steinberg module.

Using a "meataxe" program written in MAGMA, together with a MAGMA program to compute Brauer characters, we have found that $\left(\mathbb{F}_{2} \underset{A}{\otimes} P_{4}\right)_{8}$ is an extension

$$
0 \longrightarrow N^{*} \oplus T \longrightarrow\left(\mathbb{F}_{2} \otimes P_{\mathcal{A}} P_{4}\right)_{8} \longrightarrow \Lambda \oplus M \longrightarrow 0,
$$




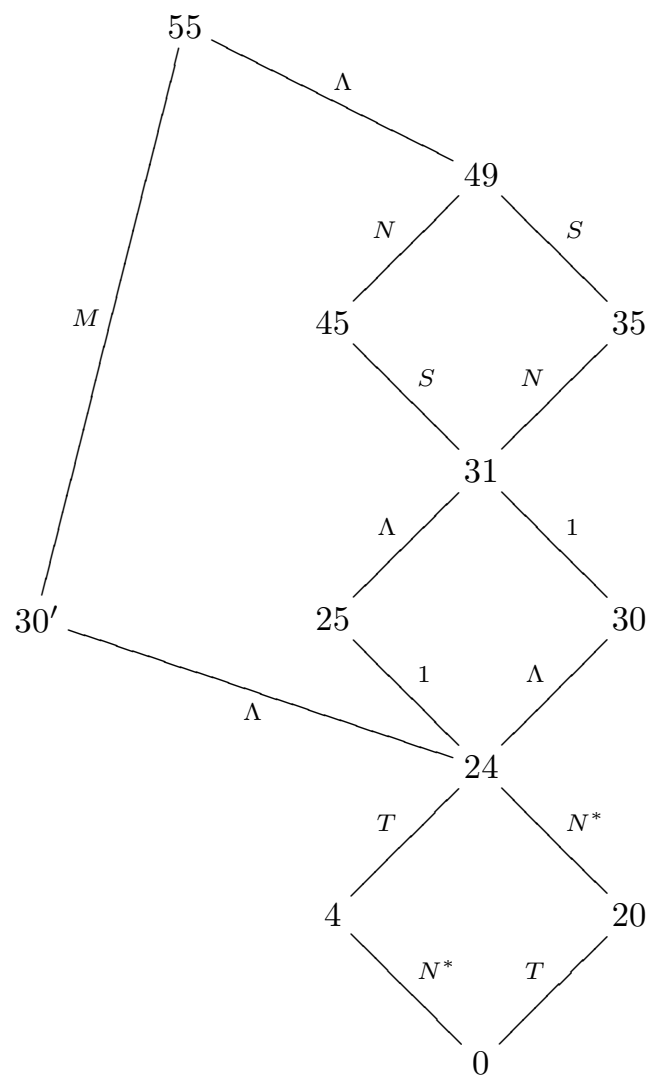

FiguRE 1. Some $G L_{4}$-submodules of $\left(\mathbb{F}_{2} \otimes P_{4}\right)_{8}$.

where the 25 -dimensional module $M$ is an extension

$$
0 \longrightarrow 1 \oplus \Lambda \longrightarrow M \longrightarrow N \oplus S \longrightarrow 0 \text {. }
$$

The corresponding lattice of submodules of $\left(\mathbb{F}_{2} \underset{\mathcal{A}}{\otimes P_{4}}\right)_{8}$ is shown in Figure 1, We name the submodules by their dimension, using a prime to distinguish the two submodules of dimension 30. We label the edges by the corresponding quotient module. In it, intersections are shown, but sums are omitted for clarity. That is, the intersection of the submodules $30^{\prime}$ and 35 is the submodule 24 , but the sum of $30^{\prime}$ and 35 (a submodule of dimension 41) is not shown. The two extensions above can be seen in the lattice, in the sense that, for example, the submodule of dimension 24 is the direct sum of the submodules of dimensions 4 and 20, since their intersection is trivial. Further, the quotient of 55 by 24 is the direct sum of the quotients of $30^{\prime}$ by 24 and of 49 by 24 .

The generators for these submodules are provided by the same computer program used to find this decomposition and are listed below. When all the monomials in one of the seven families listed in Proposition 4.1 appear, we simply write the name of the family, so that, for example, all the monomials in family $A$ are in the submodule 
of dimension 20. Also, recall the element

$$
s_{G}=(4,2,1,1)+(4,1,2,1)+(1,4,2,1)
$$

used in the proof of Proposition 4.4. Finally note that elements which form bases for the subquotients can be read off by comparing these lists of generators. For example, the quotient of the module 30 by the submodule 24 is $\Lambda$, and the elements of family $D$ generate it.

$$
\begin{aligned}
4: & (6,1,1,0)+(1,6,1,0)+(1,1,6,0),(6,1,0,1)+(1,6,0,1)+(1,1,0,6), \\
& (6,0,1,1)+(1,0,6,1)+(1,0,1,6),(0,6,1,1)+(0,1,6,1)+(0,1,1,6) . \\
20: & (A),(6,1,1,0)+(1,1,6,0),(6,1,0,1)+(1,1,0,6),(6,0,1,1)+(1,0,1,6), \\
& (1,6,1,0)+(1,1,6,0),(1,6,0,1)+(1,1,0,6),(1,0,6,1)+(1,0,1,6), \\
& (0,6,1,1)+(0,1,1,6),(0,1,6,1)+(0,1,1,6) . \\
24: & (A) \text { and }(C) . \\
25: & (A),(C), \text { and } s_{G} . \\
30: & (A),(C) \text {, and }(D) . \\
30 ': & (A),(C) \text { and }(5,1,1,1)+(1,5,1,1)+s_{G}+(3,3,1,1), \\
& (5,1,1,1)+(1,1,5,1)+s_{G}+(3,1,3,1),(5,1,1,1)+(1,1,1,5)+s_{G}+(3,1,1,3), \\
& (1,5,1,1)+(1,1,5,1)+s_{G}+(1,3,3,1),(1,5,1,1)+(1,1,1,5)+s_{G}+(1,3,1,3), \\
& (1,1,5,1)+(1,1,1,5)+s_{G}+(1,1,3,3) . \\
31: & (A),(C),(D) \text { and } s_{G} . \\
35: & (A),(C),(D), s_{G} \text { and } \\
& (5,2,1,0)+(5,2,0,1)+(5,0,2,1)+(5,1,1,1), \\
& (2,5,1,0)+(2,5,0,1)+(0,5,2,1)+(1,5,1,1), \\
& (2,1,5,0)+(2,0,5,1)+(0,2,5,1)+(1,1,5,1), \\
& (2,1,0,5)+(2,0,1,5)+(0,2,1,5)+(1,1,1,5) . \\
45: & (A),(C),(D),(E) \text { and }(G) . \\
49: & (A),(C),(D),(E),(F) \text { and }(G) . \\
&
\end{aligned}
$$

\section{ACKNOWLEDGMENT}

The research was in progress during the second named author's visit to the IHES (France) and the third named author's visit to Wayne State University, Detroit (Michigan) in the academic year 2001-2002.

The second named author thanks Lionel Schwartz for his support and encouragement.

The third named author is grateful to Daniel Frohardt, David Handel, Lowell Hansen, John Klein, Charles McGibbon, Claude Schochet and all colleagues at the Department of Mathematics, Wayne State University, for their hospitality and for the warm working atmosphere.

The authors express their hearty thanks to Trần N. Nam for helpful discussions.

\section{REFERENCES}

[1] J. M. Boardman, Modular representations on the homology of powers of real projective space, Algebraic Topology: Oaxtepec 1991, M. C. Tangora (ed.), Contemp. Math. 146 (1993), 4970. MR 95a:55041

[2] E. Brown and F. P. Peterson, $H^{*}(M O)$ as an algebra over the Steenrod algebra, Notas Mat. Simpos. 1 (1975), 11-21. MR 86b:55001

[3] M. C. Crabb and J. R. Hubbuck, Representations of the homology of BV and the Steenrod algebra II, Algebraic Topology: New Trends in Localization and Periodicity (Sant Feliu de Guíxols, 1994; C. Broto et al., eds.), Progr. Math. 136, Birkhäuser, 1996, 143-154. MR 97h:55018 
[4] Nguyễn H. V. Hưng, Spherical classes and the algebraic transfer, Trans. Amer. Math. Soc. 349 (1997), 3893-3910. MR 98e:55020

[5] C. Jansen, K. Lux, R. Parker, R. Wilson, An atlas of Brauer characters (Appendix 2 by T. Breuer and S. Norton). London Mathematical Society Monographs. New Series, 11. Oxford Science Publications. The Clarendon Press, Oxford University Press, New York, 1995. MR 96k:20016

[6] M. Kameko, Products of projective spaces as Steenrod modules, Thesis, Johns Hopkins University 1990.

[7] M. Kameko, Generators of the cohomology of $B V_{3}$, J. Math. Kyoto Univ. 38 (1998), 587-593. MR 2000b:55015

[8] W. H. Lin, Some differentials in Adams spectral sequence for spheres, Trans. Amer. Math. Soc., to appear.

[9] W. H. Lin and M. Mahowald, The Adams spectral sequence for Minami's theorem, Contemp. Math. 220 (1998), 143-177. MR 99f:55023

[10] A. Liulevicius, The factorization of cyclic reduced powers by secondary cohomology operations, Mem. Amer. Math. Soc. 42 (1962). MR 31:6226

[11] J. Peter May, The cohomology of restricted Lie algebras and of Hopf algebras; applications to the Steenrod algebra, Ph. D. thesis, Princeton University, 1964.

[12] J. Milnor and J. Moore, On the structure of Hopf algebras, Ann. of Math. 81 (1965), 211-264. MR 30:4259

[13] N. Minami, The iterated transfer analogue of the new doomsday conjecture, Trans. Amer. Math. Soc. 351 (1999), 2325-2351. MR 99i:55023

[14] W. M. Singer, The transfer in homological algebra, Math. Zeit. 202 (1989), 493-523. MR 90i: 55035

[15] R. M. W. Wood, Steenrod squares of polynomials and the Peterson conjecture, Math. Proc. Cambridge Phil. Soc. 105 (1989), 307-309. MR 90a:55030

Department of Mathematics, Wayne State University, 656 W. Kirby Street, Detroit, MiCHIGAN 48202

E-mail address: rrb@math.wayne.edu

Université de Lille I, UFR de Mathématiques, UMR 8524, 59655 Villeneuve D'AscQ CÉdex, France

E-mail address: Minh-Ha.Le@math.univ-lille1.fr

Department of Mathematics, Vietnam National University, 334 Nguyên Trãi Street, HANOI, VIETNAM

E-mail address: nhvhung@vnu.edu.vn 